\title{
Inhibition of Epithelial Cell Death by Bcl-2 Improved Chronic Colitis in IL-10 KO Mice
}

Tsunekazu Mizushima, ${ }^{*}$ Satoko Arakawa, ${ }^{\dagger}$ Yasuaki Sanada,${ }^{\dagger}$ Ikuyo Yoshino, ${ }^{\dagger}$ Dai Miyazaki, ${ }^{\dagger}$ Hayato Urushima, ${ }^{*}$ Yoshihide Tsujimoto, ${ }^{\S}$ Toshinori Ito, ${ }^{*}$ and Shigeomi Shimizu

From the Departments of Surgery* and Medical Genetics, ${ }^{\S}$ Osaka University Graduate School of Medicine, Osaka; the Department of Pathological Cell Biology, ${ }^{\dagger}$ Medical Research Institute, Tokyo Medical and Dental University, Tokyo; and the Department of Pharmacy, ${ }^{\ddagger}$ Kure Medical Center/Chugoku Cancer Center, Hiroshima, Japan

\author{
Accepted for publication \\ August 7, 2013. \\ Address correspondence to \\ Tsunekazu Mizushima, M.D., \\ Ph.D., Department of Surgery, \\ Osaka University Graduate \\ School of Medicine, Yamada- \\ oka 2-2, Suita, Osaka 565-0871, \\ Japan, or Shigeomi Shimizu, \\ M.D., Ph.D., Department of \\ Pathological Cell Biology, \\ Medical Research Institute, \\ Tokyo Medical and Dental \\ University, Yushima 1-5-45, \\ Bunkyo-ku, Tokyo 113-8510, \\ Japan. E-mail: tmizushima@ \\ gesurg.med.osaka-u.ac.jp or \\ shimizu.pcb@mri.tmd.ac.jp.
}

IL-10-deficient mice spontaneously develop intestinal inflammation, which has many similarities to Crohn's disease. Several reports suggest that epithelial cell death may increase the severity of colitis; however, decisive evidence is lacking. In the present report, we addressed whether and how epithelial cell death plays a role in the development of chronic colitis. We first examined the morphological characteristics of intestines of IL-10-deficient mice and found two forms of epithelial cell death (typical apoptosis and necrosis-like cell death) in colitis. To elucidate the pathological roles of epithelial cell death, we crossbred IL-10-deficient knockout mice with Bcl-2 transgenic mice, in which the anti-apoptosis protein Bcl-2 was overexpressed in intestinal epithelial cells, but not in immune cells. Epithelial cell-specific Bcl-2 protected IL-10 deficiency-induced colitis and markedly reduced their symptoms. Interestingly, morphological analysis revealed that Bcl-2 suppressed apoptosis and necrosis-like cell death, and better maintained mucosal barrier in IL-10-deficient mice. From the immunological aspect, Bcl-2 did not alter the activation of T-helper cell 1 but inhibited the growth of T-helper cell 17, suggesting that mucosal integrity may control the immune responses. These results provide genetic evidence demonstrating that epithelial cell death is crucial for the development of chronic colitis. (Am J Pathol 2013, 183: 1936-1944; http://dx.doi.org/ 10.1016/j.ajpath.2013.08.012)
Crohn's disease (CD) and ulcerative colitis are two major chronic inflammatory bowel diseases (IBDs) of the gastrointestinal tract in humans. The pathogenesis of IBD has not yet been fully elucidated because of the involvement of multifactorial interactions between genetic factors and environmental triggers, ${ }^{1}$ but is thought to result from the inappropriate and ongoing activation of the mucosal immune system driven by a breakdown of immunological tolerance to certain exogenous antigens or luminal flora. ${ }^{2,3}$ The healthy intestinal epithelium provides a physical and immunological barrier that is relatively impermeable to luminal constituents, such as bacteria, bacterial products, and food antigens, which may be capable of initiating and sustaining physiological and pathological inflammation in the gut. Therefore, once an intestinal epithelial cell is damaged, the aberrant immune response is likely facilitated by defects of barrier function of the intestinal epithelium. ${ }^{4}$ Puma, a proapoptotic protein, plays a role in epithelial cell death in acute drug-induced colitis. ${ }^{5,6}$ In chronic colitis, however, definite data for proving the significance of epithelial cell death are lacking. There should be some differences in pathophysiological characteristics, particularly in the role of cell death, between acute drug-induced colitis and chronic genetic colitis.

Several animal models of IBD have been developed by genetic engineering. Among them, the IL-10-deficient

\footnotetext{
Supported in part by a Grant-in-Aid for Scientific Research on Innovative Areas, a Grant-in-Aid for Scientific Research (S), and a Grant-in-Aid for Challenging Exploratory Research from the Ministry of Education, Culture, Sports, Science and Technology of Japan and grants from the Program for Promotion of Fundamental Studies in Health Sciences of the National Institute of Biomedical Innovation, the Research Fund of Mitsukoshi Health and Welfare Foundation, The Secom Science and Technology Foundation, The Takeda Science Foundation, The Uehara Memorial Foundation, The Sumitomo Foundation, The SENSHIN Medical Research Foundation, and The Naito Foundation.
} 
knockout (KO) mice are considered as the most appropriate model of human CD for several reasons: i) they develop spontaneous colitis with multifocal inflammatory lesions throughout the gastrointestinal tract; ii) colitis develops when the mice are bred under conventional, but not germ-free, animal care facilities; and iii) colitis is characterized by infiltration of activated CD4 ${ }^{+}$T-helper cell 1 (Th1), exclusively producing interferon (IFN)- $\gamma$ and macrophages into the lamina propria of the intestine. Therefore, we used IL-10 KO mice to examine the role of epithelial cell death.

Our strategy was to generate transgenic (Tg) mice overexpressing an anti-apoptotic protein specifically in the epithelial cells of mice and crossbreed these mice with IL-10 $\mathrm{KO}$ mice. We used Bcl-2 Tg mice, in which human BCL2 gene, a well-known anti-apoptotic gene, is overexpressed in the epithelial cells of intestines. ${ }^{7}$ Our genetic approach revealed that epithelial cell-specific Bcl-2 inhibited cell death in the intestinal epithelium, better maintained the mucosal barrier, and reduced the Th17 lymphocyte population and total number of colonic lamina propria (CLP) lymphocytes, thereby largely suppressing intestinal inflammation and prolonging the survival of IL-10 KO mice. To our knowledge, these results are the first to show a decisive conclusion indicating the important role of epithelial cell death in chronic colitis.

\section{Materials and Methods}

Mice

Breeding pairs of IL-10 KO mice (C57BL/6 background) were purchased from Jackson Laboratory (Bar Harbor, ME). C57BL/6 mice [wild type (WT)] were purchased from Japan CLEA (Tokyo, Japan). The generation of human Bcl-2 Tg mice on a C57BL/6 genetic background has been described previously. ${ }^{7,8}$ In Bcl-2 Tg mice, human Bcl-2 cDNA was directed by the regulatory sequences of the rat L-type pyruvate kinase gene that encodes a glycolytic enzyme expressed in epithelial cells of the intestines, hepatocytes, and proximal tubular cells of the kidney, but not in immune cells. ${ }^{7}$ IL-10 KO Bcl-2 Tg mice and IL-10 KO littermate mice were generated by crossbreeding. All of the mice were maintained in the specific pathogen-free animal facility at the Institute of Experimental Animal Sciences, Osaka University Medical School (Osaka, Japan), and Tokyo Medical and Dental University (Tokyo, Japan). The genotypes of mice were determined by PCR analysis of DNA isolated from tail tissue, using the following synthetic oligonucleotide murine primer pairs: IL-10, $5^{\prime}$-TAGGCGAATGTTCTTCC-3' (sense) and 5'-CAGGCAGCATAGCAGTG-3' (antisense); IL-10 sense and neomycin, 5'-CCTGCGTGCAATCCATCTTG-3'; Bcl-2 Tg, 5'-TGGCACAGGCTGTGTTT-3' (sense) and 5'-GAATTCCAGGGCATCAG-3' (antisense). All experiments were reviewed and approved by the Institutional Animal Care and Use Committee in Osaka University and Tokyo Medical and Dental University, and were conducted according to the committees' guidelines.

\section{Electron Microscopy}

Tissues were fixed by a conventional method (1.5\% paraformaldehyde $/ 3 \%$ glutaraldehyde in $0.1 \mathrm{~mol} / \mathrm{L}$ phosphate buffer at $\mathrm{pH} 7.3$, followed by an aqueous solution of $1 \%$ $\mathrm{OsO}_{4}$ ). Fixed samples were embedded in Epon 812 (TAAB Laboratories, Berks, UK); sections (70 to $80 \mathrm{~nm}$ thick) were cut and stained with uranyl acetate and lead citrate, and observed using a JEOL-1010 (JEOL, Tokyo, Japan) at $80 \mathrm{kV}$.

\section{Histological Analysis}

The colons of 16- to 20-week-old mice were divided into proximal, middle, and distal portions. Tissue was fixed in $4 \%$ paraformaldehyde in PBS for 4 hours and embedded in paraffin for the preparation of sections ( $2 \mu \mathrm{m}$ thick) that were stained with H\&E for the assessment of disease and clinical score. Histopathological alterations in the colon were semiquantified according to a modified scoring system using the following criteria: i) cellular infiltration in CLP (score, 0 to 3 ), ii) mucin depletion (score, 0 to 2 ), iii) crypt abscesses (score, 0 to 2), iv) epithelial erosion (score, 0 to 2), v) hyperemia (score, 0 to 3 ), and vi) thickness of the colonic mucosa (score, 1 to 3 ). ${ }^{9}$ Hence, the range of histopathological scores of each specimen was from 1 (no alteration) to 15 (most severe colitis) and that of each mouse was from 3 to 45 .

To evaluate epithelial apoptosis, we performed TUNEL staining using the ApopTag Plus Kit (Oncor Co, Gaithersburg, $\mathrm{MD}$ ), according to the manufacturer's protocol. The sections were counterstained with hematoxylin and then mounted. We also performed Western blot analysis as follows: the colon was excised and homogenized in isotonic buffer $[20 \mathrm{mmol} / \mathrm{L}$ potassium HEPES (pH 7.4), $10 \mathrm{mmol} / \mathrm{L} \mathrm{KCl}, 1.5 \mathrm{mmol} / \mathrm{L} \mathrm{MgCl}$, $250 \mathrm{mmol} / \mathrm{L}$ sucrose, $1 \mathrm{mmol} / \mathrm{L}$ EDTA, $0.1 \mathrm{mmol} / \mathrm{L}$ phenylmethylsulfonyl fluoride, and $1 \mathrm{mmol} / \mathrm{L}$ dithiothreitol], followed by sonication on ice and centrifugation. The amount of active caspase-3 and cleavage of inhibitor of caspase activated DNase (ICAD) were estimated by using Western blot analysis using anti-active caspase-3 monoclonal (Mab835; R\&D Co, Minneapolis, MN) and anti-ICAD polyclonal (FL-331; Santa Cruz Biotechnology, Santa Cruz, CA) antibodies, respectively.

\section{Evaluation of Mucosal Integrity}

We used a fluorescein isothiocyanate (FITC) - dextran assay to evaluate mucosal integrity. IL-10 KO Bcl-2 Tg and IL-10 KO mice were fasted, and $200 \mu \mathrm{L}$ of FITC-dextran $(2 \mathrm{mg} / \mathrm{mL}$ in saline; average molecular weight, 4400; Sigma Chemical Co, St. Louis, MO) was administered orally 12 hours later. The proximal colon was snap frozen 4 hours later, and cryostat sections $(10 \mu \mathrm{m}$ thick) were cut and observed by fluorescence microscopy. ${ }^{10}$ The amount of FITC-dextran fluorescence (area $x$ intensity) in the intraepithelial space and the extraintestinal space was measured using Imaris imaging software version 5.5 (Bitplane Scientific, Zurich, Switzerland), and the ratio of FITCdextran in the intraepithelial space/total area was calculated. 
We also assessed barrier permeability by analyzing serum concentrations of FITC-dextran. In brief, the mice were orally administered $60 \mu \mathrm{g} / 100 \mathrm{mg}$ body weight FITC-dextran, and serum was collected after 4 hours. Then, fluorescence intensity was measured using a fluorescence spectrophotometer (485 excitation/520 emission). FITC-dextran concentration was determined from standard curves generated by serial dilution of FITC-dextran.

\section{Analysis of Lymphoid Cells in CLP}

Whole CLP lymphocytes were collected from the entire colon by an enzymatic dissociation method using collagenase, as described previously. ${ }^{11}$ For CD4 single-color flow cytometry, $1 \times 10^{6}$ cells were stained with phycoerythrin-conjugated antiCD4 (RM4-5; BD Biosciences, San Jose, CA) monoclonal antibody (mAb). Negative control samples were stained with an irrelevant, isotype antibody in parallel with all experimental samples. CD4/IL-17 double-color flow cytometry was performed using a Cytofix/Cytoperm Kit (BD Biosciences), according to the manufacturer's instructions. In brief, CLP lymphocytes were first incubated with $50 \mathrm{ng} / \mathrm{mL}$ phorbol myristate acetate (Sigma Chemical Co), $5 \mu \mathrm{mol} / \mathrm{L}$ calcium ionophore (Sigma Chemical Co), and Golgistop (BD Biosciences) at $37^{\circ} \mathrm{C}$ for 4 hours. Then, the lymphocytes were stained with phycoerythrin-labeled anti-CD4 mAb for $30 \mathrm{mi}-$ nutes at $4{ }^{\circ} \mathrm{C}$. The cells were then permeabilized with Cytofix/ Cytoperm for 20 minutes at $4^{\circ} \mathrm{C}$, and intracellular IL-17 was stained with Cy5-labeled anti-IL-17A mAb (TC11-18H10.1) for 30 minutes at $4^{\circ} \mathrm{C}$. Finally, the cells were analyzed using an FACSCalibur flow cytometer (BD Biosciences).

To measure cytokine production, $1 \times 10^{6}$ CLP lymphocytes $/ \mathrm{mL}$ were seeded in anti-CD3 $\varepsilon$ antibody-coated dishes (clone145-2C11; Becton Dickinson) and cultured with $1 \mu \mathrm{g} /$ $\mathrm{mL}$ soluble anti-CD28 (clone 37.51; Becton Dickinson) for 48 hours. IFN- $\gamma$ and IL-4 levels produced were determined with commercially available specific ELISA kits using duopaired murine cytokines, as per the manufacturer's recommendations (Becton Dickinson). Optical densities were measured using a MR5000 ELISA reader (Dynatech Technologies, Chantilly, VA) at a wavelength of $450 \mathrm{~nm}$.

\section{Statistical Analysis}

Data are presented as the means \pm SEM. Data were analyzed using an unpaired two-tailed $t$-test or repeatedmeasures analysis of variance. An associated probability $(P$ value) of $<0.05$ was considered significant.

\section{Results}

Ultrastructural Analysis of Colon Tissue from IL-10 K0 Mice

IL-10 KO mice, genetic models of human IBD, spontaneously develop enterocolitis by 2 to 3 months of age. ${ }^{12-14}$ To clarify the pathogenesis of colitis, we first examined the mice for morphological abnormalities. The noticeable macroscopical abnormalities in the colon of the IL-10 KO mice appeared as multifocal inflammatory lesions with epithelial hyperplasia and aphthous ulcers throughout the intestinal tract (Figure 1, A and B). In the ulcer, we observed shedding epithelial cells, exposure of the submucosa, and massive accumulation of neutrophils (Figure 1, B and C). Some neutrophils leaked out into the gut lumen (Figure 1, B and C). The same section examined by transmission electron microscopy (EM) revealed prominent activation of Gram-negative bacteria that had penetrated deep into the submucosal area. Many bacteria had invaded into and proliferated within some epithelial cells (Figure 1, B-D, and Supplemental Figure S1C). The EM analysis also revealed induction of two forms of epithelial cell death: typical apoptosis (Figure 1E and Supplemental Figure S1D) and necrosis-like cell death (Figure 1, B, C, and F, and Supplemental Figure S1E). The typical apoptotic cells lost their polarity and exhibited condensed chromatin with mild mitochondrial swelling (Figure 1E). Bacteria were never observed in the apoptotic cells. In contrast, necrosis-like cells showed a clear nucleoplasm, probably because of the extraordinary DNA digestion and accompanied severe mitochondrial swelling (Figure 1, B and F). Cells in the end stage of the necrosis-like process were deformed by rupture of their plasma membranes (Figure 1G). All of these cells were severely invaded by bacteria, and their morphological characteristics were clearly distinguished from those of apoptotic cells. Furthermore, immunofluorescence analysis of the necrosis-like cells revealed that total caspase-3, but not active caspase-3, was present in these cells (Supplemental Figure S2, B and C), and cytochrome $c$ was still retained in mitochondria (Supplemental Figure S2F), suggesting that the apoptosis machinery was not activated in these necrosis-like cells. Thus, apoptosis and necrosis-like cell death were induced in chronic colitis. Massive accumulation of plasma cells with elongated ERs was observed in the lamina propria (indicating abundant antibody synthesis) (Figure $1 \mathrm{H}$ ). All these phenotypes were considerably similar to those characteristic of human $\mathrm{CD} .{ }^{15}$

\section{Generation of IL-10 K0/Bcl-2 Transgenic Mice}

To understand the significance of epithelial cell death in chronic enterocolitis development, we attempted to inhibit epithelial cell death by genetically overexpressing the antiapoptotic protein, Bcl-2. ${ }^{7}$ We mated IL-10 $\mathrm{KO}$ mice with human Bcl-2 $\mathrm{Tg}$ mice and generated $\mathrm{IL}-10 \mathrm{KO} / \mathrm{Bcl}-2 \mathrm{Tg}$ mice. In the Bcl-2 $\mathrm{Tg}$ mice, human Bcl-2 cDNA was expressed mainly in the epithelial cells of the intestines, but not in immune cells. ${ }^{7}$ We backcrossed 10 generations to avoid the influence of genetic background. We confirmed human Bcl-2 expression in the colonic epithelium of the Bcl-2 $\mathrm{Tg}$ mice by using Western blot analysis (Supplemental Figure S3A) and immunohistochemistry (IHC) (Supplemental Figure S3, B and C). We also 

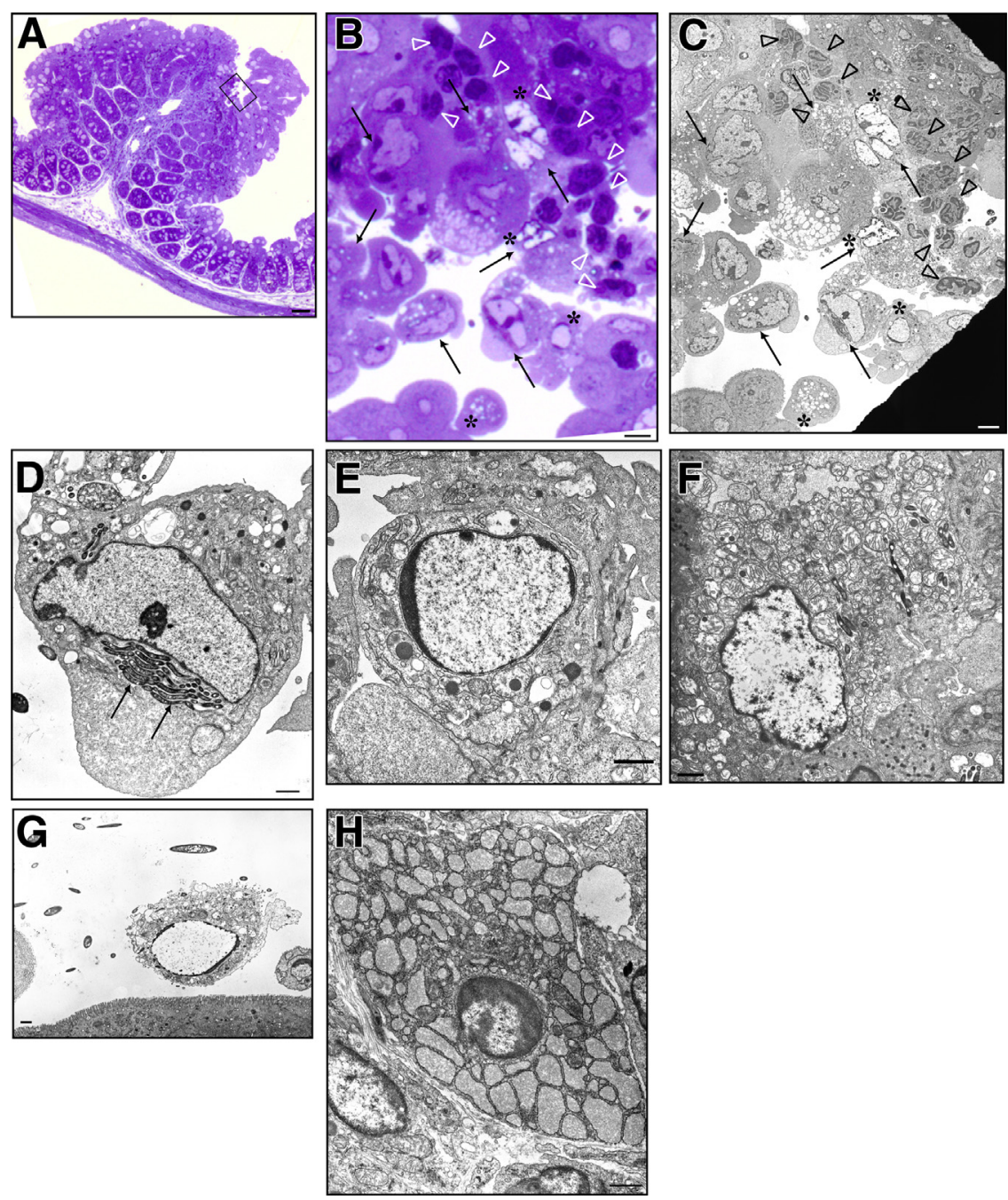

Figure 1 Morphological analysis of the colons of IL-10 K0 mice. A-C: Colonic section showing cell death in IL-10 K0 mice at 16 weeks of age. A: Lowmagnification image stained with toluidine blue. B and C: Light and electron microscopic analysis. Colonic sections (boxed in A) were fixed as described in Materials and Methods and then stained with toluidine blue (B). The serial section was examined by EM (C). Note bacteria-invaded epithelial cells (arrows), accumulation of neutrophils (arrowheads), and dead epithelial cells (asterisks). A magnification is shown in Supplemental Figure S1. D: Representative image of a bacteriainvaded epithelial cell. Gram-negative bacteria (arrows) proliferated next to the nucleus. E: Representative image of an apoptotic epithelial cell. $\mathbf{F}$ and $\mathbf{G}$ : Representative images of epithelial necrosis-like cells. Necrosis-like cells showing clear nucleoplasms with severe mitochondrial swelling (F). Ruptured end-stage cells with bacteria spewing from them $(\mathbf{G})$. H: Representative image of a plasma cell. Endoplasmic reticulum (ER) is prominently elongated. Scale bars: $50 \mu \mathrm{m}$ (A and C); $1 \mu \mathrm{m}$ (D, E, and $\mathbf{G}) ; 5 \mu \mathrm{m}(\mathbf{H})$. examined whether overexpressed Bcl-2 may have sufficient anti-apoptotic activity in intestinal epithelial cells. When WT mice were exposed to X-ray irradiation (15 Gy) and sacrificed after 4 days, their intestines were severely damaged with denudation of the crypt and villus system (Supplemental Figure S3D), and many epithelial cells were positive for TUNEL staining (Supplemental Figure S3E). In contrast, the intestine of the $\mathrm{Bcl}-2 \mathrm{Tg}$ mice appeared normal (Supplemental Figure S3G), and only a few TUNEL-positive cells were observed in the columnar epithelium (Supplemental Figure $\mathrm{S} 3 \mathrm{H}$ ), even after irradiation. Unlike epithelial cells, the lymphocytes in intestinal lymphoid follicles, where transgenic Bcl-2 was not expressed (Supplemental Figure S3A), were equally susceptible to cell death by irradiation (Supplemental Figure S3, F and I). These data indicate that the transgenic Bcl-2 had significant anti-apoptotic activity in intestinal epithelial cells but not in immune cells. Epithelial Bcl-2 expression did not alter basic intestinal physiological characteristics, including epithelial cell proliferation (Supplemental Figure S4, A and B) and basal transepithelial permeability (Supplemental Figure S4, C and D). Furthermore, the IL-10 KO and IL-10 KO/Bcl-2 Tg mice had comparable commensal bacteria (Supplemental Figure S5). Therefore, by comparing the IL-10 KO/Bcl-2 Tg mice with their IL-10 KO littermates, we could determine whether epithelial cell death was responsible for the pathogenesis of chronic colitis.

\section{Suppression of IL-10 Deficiency-Induced Colitis by Expression of $\mathrm{BCl}-2$ in Intestinal Epithelial Cells}

IL-10 KO mice are born healthy, but they gradually develop a wasting syndrome with associated rectal prolapse, chronic diarrhea, diminished weight gain, and hunched posture from 8 to 16 weeks of age. ${ }^{12}$ Compared with IL-10 KO mice, the IL-10 KO/Bcl-2 Tg mice gained more weight, maintaining it nearly in the normal range until 20 weeks of age (Figure 2, A and B). After 24 weeks, their weights were less than those of WT mice but higher than those of IL-10 KO mice (Figure 2B). Almost all of the IL-10 KO/Bcl-2 Tg mice remained healthy until 20 weeks of age. Rectal prolapse was observed after 5 weeks of age in IL-10 KO mice, whereas it was not observed until 12 weeks of age in the IL-10 KO/ Bcl-2 Tg mice (Figure 2, C and D). The incidence of 


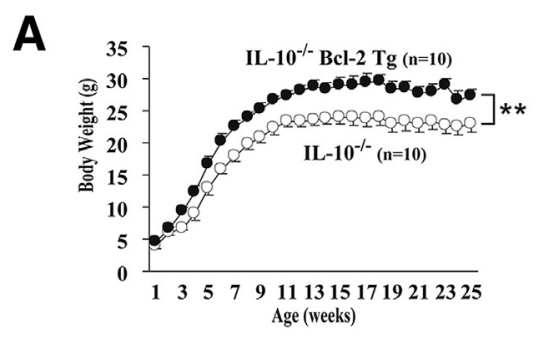

B

C

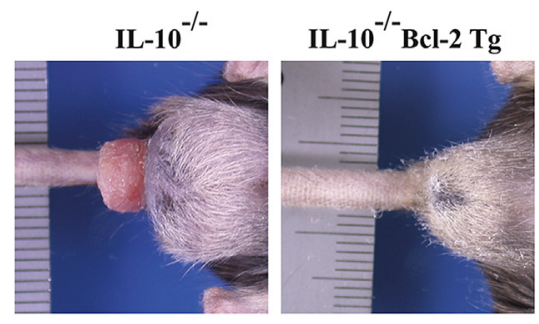

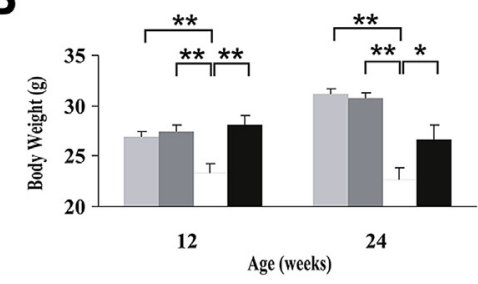

D

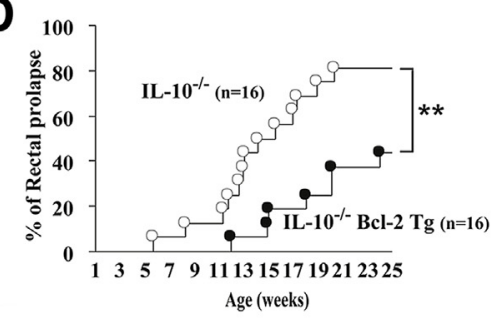

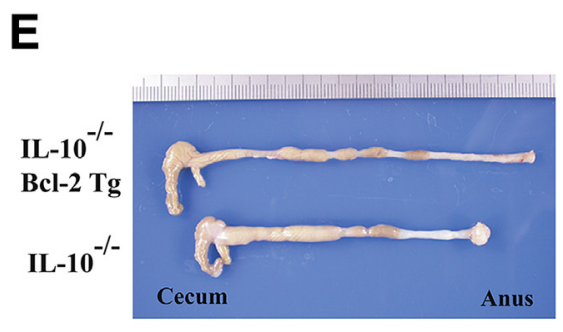

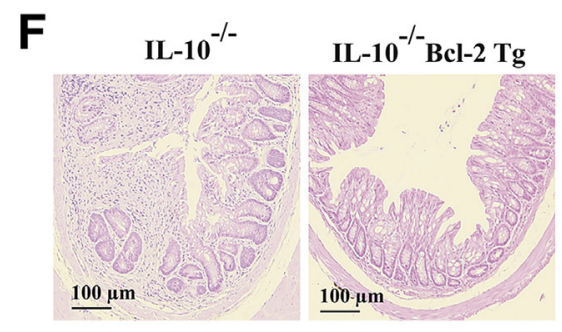

Figure 2 Partial inhibition of IL-10 deficiencyinduced colitis by expression of Bcl-2 in intestinal epithelial cells. A and B: Partial inhibition of body weight loss by $\mathrm{BCl}-2$ expression in the intestinal epithelial cells of IL-10 KO mice. The body weights of IL-10 KO (white symbols), IL-10 K0/Bcl-2 Tg (black symbols), WT (light gray symbols), and Bcl$2 \mathrm{Tg}$ (dark gray symbols) mice at the indicated ages are shown. The data are expressed as means $\pm \operatorname{SEM}(n=10)$. C and D: Partial inhibition of rectal prolapse by epithelial Bcl-2 expression in IL$10 \mathrm{KO}$ mice. Representative images of the anus of IL-10 K0 mice and IL-10 K0/Bcl-2 Tg mice at 18 weeks of age (C). D: The incidence of rectal prolapse of IL-10 K0 mice (white symbols) and IL-10 $\mathrm{KO} / \mathrm{Bcl}-2 \mathrm{Tg}$ mice (black symbols) at the indicated ages (weeks). Data are analyzed by the KaplanMeier method as determined by log-rank test (Mantel-Cox-Savage). E-G: Partial inhibition of colitis in IL-10 K0 mice by epithelial Bcl-2. E: Representative image of colon tissue from IL-10 $\mathrm{K} 0$ mice and IL-10 K0/Bcl-2 Tg mice at 16 weeks of age. The colons of IL-10 KO mice were short and thick, whereas those of IL-10 KO/Bcl-2 Tg mice looked nearly normal. F: Histological sections of the colon from IL-10 K0 mice and IL-10 K0/Bcl-2 $\mathrm{Tg}$ mice at 16 weeks of age, stained with H\&E. Colonic sections from IL-10 K0 mice had inflammatory infiltration in the lamina propria and crypt damage, whereas those from IL-10 KO/Bcl-2 Tg mice appeared to be nearly normal. G: Histological scoring of the colons of IL-10 K0 and IL-10 KO/ $\mathrm{Bcl}-2 \mathrm{Tg}$ mice at 16 to 20 weeks of age. The histological score of IL-10 $\mathrm{KO} / \mathrm{Bcl}-2 \mathrm{Tg}$ mice (8.6) was significantly lower than that of IL-10 KO mice (15.5). ${ }^{*} P<0.05,{ }^{*} P<0.01$. The data represent means \pm SEM. rectal prolapse was significantly reduced by Bcl-2 expression $(81.3 \%$ in $\mathrm{IL}-10 \mathrm{KO}$ versus $43.8 \%$ in $\mathrm{IL}-10 \mathrm{KO} / \mathrm{Bcl}-2$ $\mathrm{Tg}$ at 24 weeks of age) (Figure 2D). These results indicated that the epithelial expression of $\mathrm{Bcl}-2$ diminished the clinical features of colitis in IL-10 KO mice. Consistent with the clinical findings, at 16 weeks of age, the colons of IL-10 KO mice became short and had thicker walls, whereas those of IL-10 KO/Bcl-2 Tg mice appeared nearly normal (Figure 2E). Histologically, the colons of IL-10 KO/Bcl-2 $\mathrm{Tg}$ mice exhibited less-damaged epithelia and less infiltration of inflammatory cells than in IL-10 KO mice (Figure 2F). These findings were graded using the criteria for histological grading of colitis, as described in Materials and Methods. ${ }^{9}$ The histological scores of the IL-10 KO/Bcl$2 \mathrm{Tg}$ mice were lower than those of IL-10 KO mice ( 7.8 in IL-10 KO/Bcl-2 Tg versus 15.5 in IL-10 KO) (Figure 2G). The combined clinical and histological results demonstrated that transgenic $\mathrm{Bcl}-2$ expression in the epithelial cells resulted in less severe colitis in IL-10 KO mice, probably by reducing the epithelial cell death.

Inhibition of Epithelial Cell Death and Maintenance of Mucosal Permeability by Epithelial Bcl-2 in IL-10 K0 Mice

To confirm that Bcl-2 effectively inhibited colonic epithelial cell death, we first performed TUNEL analysis of specimens from IL-10 KO and IL-10 KO/Bcl-2 Tg mice. As shown in Figure 3, A and C, TUNEL-positive cells were numerous in the colonic epithelia of IL-10 KO mice but were markedly less numerous in IL-10 KO/Bcl-2 Tg mice (Figure 3, B and C). The rate of TUNEL-positive cells (approximately 14.5 cells per 100 crypts) in IL-10 KO mice was the same as that of apoptotic cells by EM, suggesting that apoptotic cells, but not necrosis-like cells, were stained by TUNEL assay. These results indicated that epithelial cell apoptosis was 

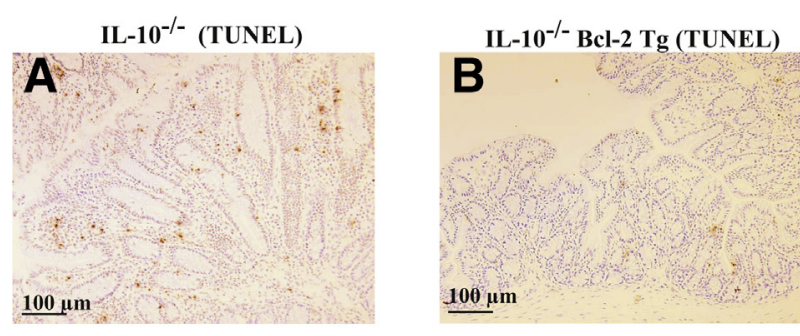

C
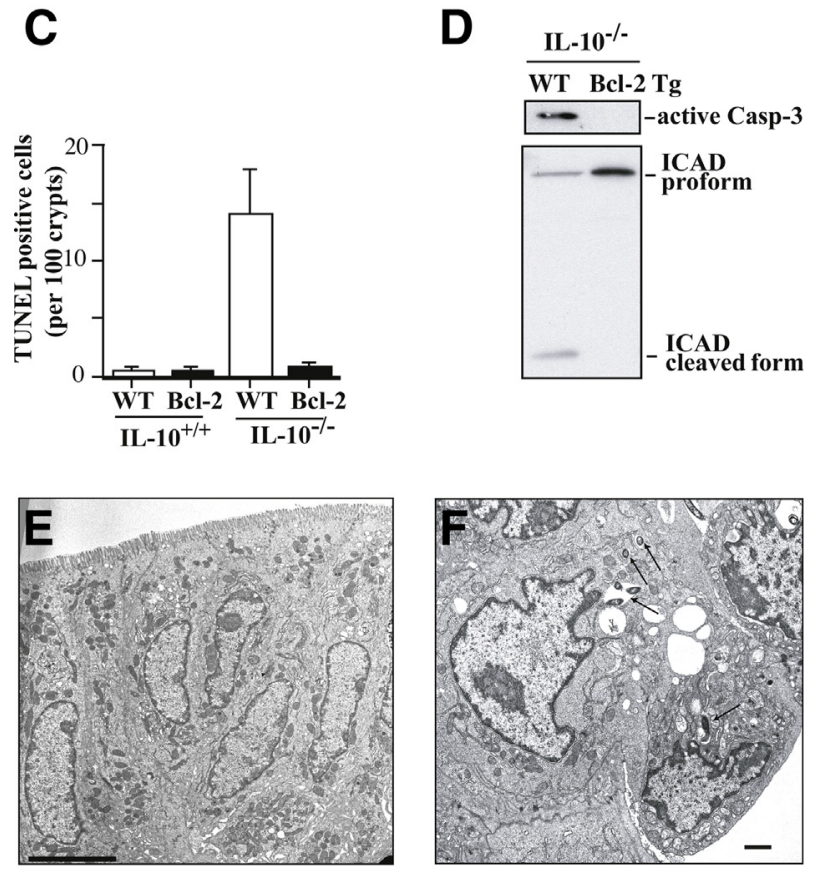

Figure 3 Inhibition of epithelial cell death by epithelial Bcl-2 in IL-10 K0 mice. A-D: Inhibition of epithelial cell apoptosis by epithelial Bcl-2. A-C: Colons from the indicated mice at 16 weeks of age were frozen, and apoptotic cells were visualized by the TUNEL method. Representative images are shown. TUNEL-positive cells were stained brown. The number of TUNEL-positive cells per 100 crypts is shown in C. D: Equivalent segments of colon were excised and homogenized. The homogenates were subjected to Western blot analysis with anti-ICAD and anti-active caspase- 3 antibodies. $\mathbf{E}$ and $\mathbf{F}$ : Representative images of the colons of IL-10 K0/Bcl-2 Tg mice at 16 weeks of age. E: There were only a few apoptotic and necrosis-like cells. F: Bacteria-invaded cells continued to be healthy and suppressed bacterial proliferation. Arrows indicate Gram-negative bacteria. Scale bars: $10 \mu \mathrm{m}(\mathbf{E}) ; 1 \mu \mathrm{m}(\mathbf{F})$.

decreased by the presence of Bcl-2. This conclusion was supported by the findings that apoptosis-associated cleavage of ICAD and activation of caspase-3 were absent in the intestines of IL-10 KO/Bcl-2 Tg mice (Figure 3D).

We next performed EM analysis to further examine the role of Bcl-2 on epithelial cell death. We observed numerous cells undergoing typical apoptosis and necrosis-like death in the colons of IL-10 KO mice (Figure 1C). In contrast, there were only a few apoptotic and necrosis-like cells in IL-10 KO/Bcl-2 Tg mice (Figure 3E). We were surprised because $\mathrm{Bcl}-2$ protected apoptosis and necrosislike cell death, although the precise mechanism is unknown (see Discussion). Interestingly, some epithelial cells in the colon of IL-10 KO/Bcl-2 Tg mice contained bacteria, but, unlike IL-10 KO mice, these cells looked healthy, containing a limited number of bacteria (Figure 3F).
In the pathogenesis of Crohn's disease, excessive epithelial cell death may destroy the barrier function against bacteria (or bacterial products) and may exacerbate the colitis. Therefore, we next addressed whether Bcl-2 expression effectively provided a barrier relatively impermeable to intestinal constituents. To this end, we fed FITC-dextran to the mice, excised their colons after 4 hours, and examined them by fluorescent microscopy. We found that FITCdextran had markedly infiltrated into the submucosa of IL-10 KO mice (Figure 4, A and B), but not those of IL-10 $\mathrm{KO} / \mathrm{Bcl}-2 \mathrm{Tg}$ mice (Figure 4, $\mathrm{B}$ and $\mathrm{C}$ ) or WT mice (Figure 4B). Consistently, the extent of serum FITC-dextran that crossed the epithelial barrier of IL-10 KO mice was higher than that of WT mice or IL-10 KO/Bcl-2 Tg mice (Figure 4D). These data indicated that Bcl-2 had maintained the colonic mucosal barrier, possibly by preventing epithelial cell death. EM analysis also showed that healthy epithelial cells were lined to prevent bacterial invasion (Figure 3E).

\section{Analysis of Mucosal Lymphocytes}

Colitis in IL-10 KO mice is mediated by infiltration of activated $\mathrm{CD}^{+} \mathrm{T}$ lymphocytes and macrophages into the CLP; these cells produce Th1 and Th17 cytokines. ${ }^{13,16-18}$ Therefore, we next examined whether epithelial Bcl-2 expression influenced the immune responses that characterize the IL-10 KO mice. We collected whole CLP lymphocytes from 8- to 12 -week-old mice whose symptoms had not yet appeared. The population of $\mathrm{CD}^{+}{ }^{+} \mathrm{T}$ lymphocytes in CLP of the IL-10 KO mice was considerably higher than that in CLP of the WT mice. Despite suppression of colitis in the IL-10 KO/Bcl-2 $\mathrm{Tg}$ mice, their $\mathrm{CD}^{+}{ }^{+} \mathrm{T}$ lymphocyte population was the same as that of the IL-10

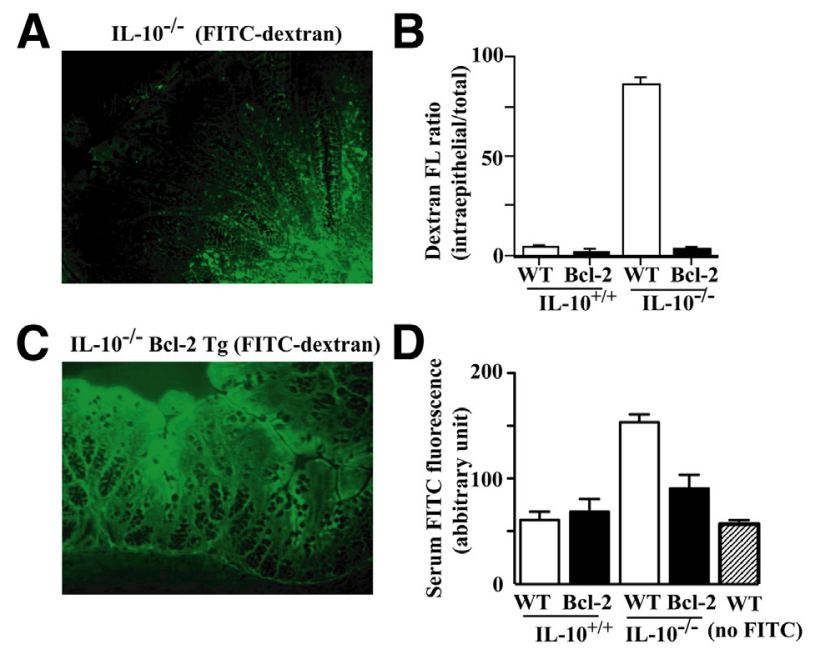

Figure 4 Maintenance of mucosal permeability by epithelial $\mathrm{Bcl}-2$ in IL-10 K0 mice. Colons from the indicated mice at 16 weeks of age that had been administered oral FITC-dextran were frozen and examined using a fluorescence microscope. Representative images are shown in $\mathbf{A}$ and $\mathbf{C}$. The extent of infiltrated FITC-dextran was calculated from the ratio of the intraepithelial fluorescence to extraepithelial fluorescence (B). D: The extent of serum FITC-dextran was measured as described in Materials and Methods. 
A

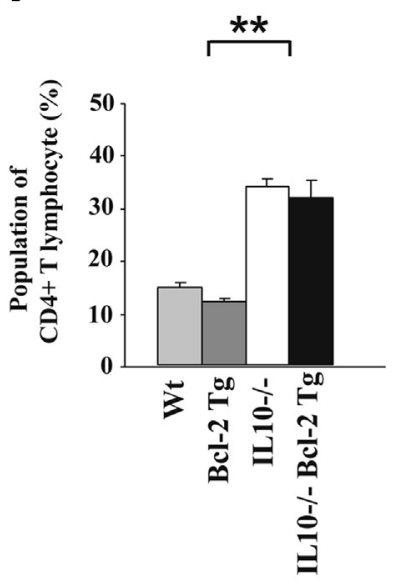

B

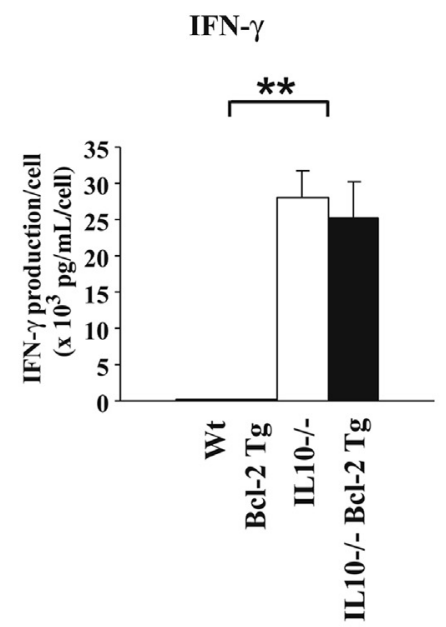

C
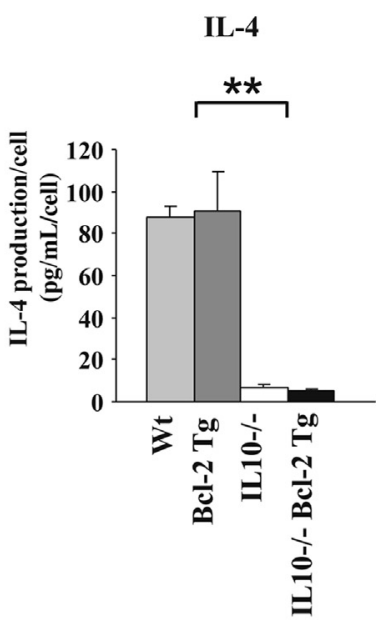

D

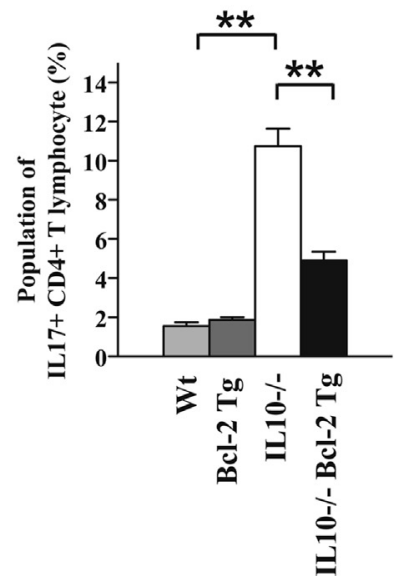

E
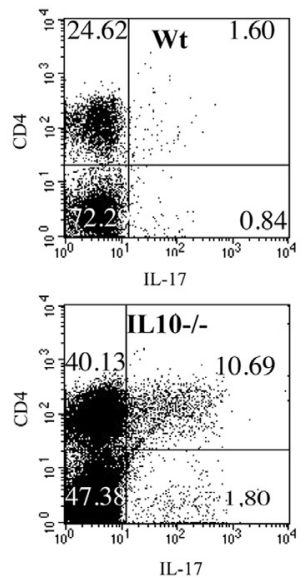

$\mathbf{F}$
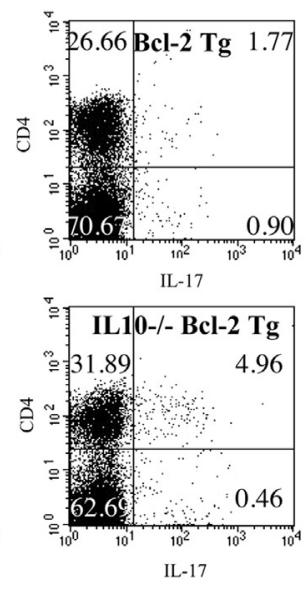

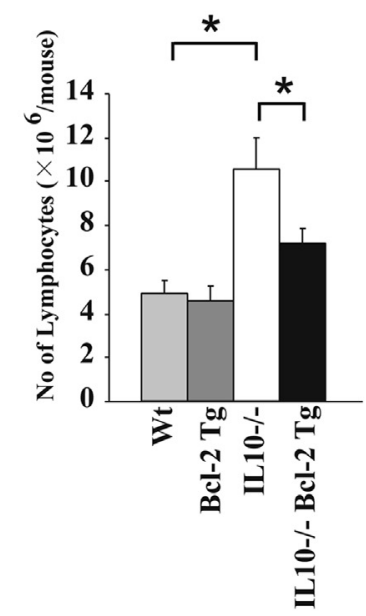

Figure 5 Analysis of cytokine profiles of mucosal lymphocytes. A: The population of CD4 ${ }^{+} T$ lymphocytes in the CLP. Whole CLP lymphocytes from the entire colon were stained with phycoerythrin-conjugated anti-CD4 monoclonal antibody, and the population of CD4 ${ }^{+} \mathrm{T}$ lymphocytes/total CLP lymphocytes was analyzed. The population of $\mathrm{CD4}^{+} \mathrm{T}$ lymphocytes in IL-10 knockout mice was higher than that in WT mice, irrespective of the presence of Bcl-2. Data represent means \pm SEM from more than four different experiments. Levels of IFN- $\gamma$ (Th1 cytokine) (B) and IL-4 (Th2 cytokine) (C) released from the CLP lymphocytes of each mouse. The CLP lymphocytes were seeded in anti-CD3 $\varepsilon$ antibody-coated dishes and cultured with $1 \mu \mathrm{g} / \mathrm{mL}$ soluble anti-CD28 for 48 hours. IFN- $\gamma$ and IL-4 levels produced were examined. Data represent means \pm SEM from more than four different experiments. D: The population of Th17 lymphocytes in CLP (D). The lymphocytes were stained with anti-IL17 and anti-CD4 antibodies and analyzed by flow cytometry (E). Data represent means \pm SEM from more than four different experiments. F: The number of lymphocytes in CLP. Single-cell suspensions of CLP were collected from the entire colon and their numbers were counted. ${ }^{*} P<0.05,{ }^{* *} P<0.01$.

KO mice (Figure 5A). To confirm this result, we purified lymphocytes from CLP of these mice and measured cytokine production. As shown in Figure $5 \mathrm{~B}$, production of IFN- $\gamma$, a Th1 cytokine, was up-regulated in the CLP lymphocytes of the IL-10 KO mice and IL-10 KO/Bcl-2 Tg mice. Consistent with these results, production of IL-4, a Th2 cytokine, was down-regulated in the CLP lymphocytes of these mice (Figure 5C). These results indicate that the lymphocyte cytokine profile was shifted to the Th1 type irrespective of Bcl-2 expression levels. Therefore, the Th1dominant phenotype seemed to be a primary event in IL-10 deficiency. Unlike Th1, the population of Th17 lymphocytes in CLP of the IL-10 KO mice was higher than that of the WT mice, but this population was markedly reduced in the IL-10 KO/Bcl-2 Tg mice (Figure 5, D and E). Thus,
Bcl-2-dependent mucosal integrity may control Th17 cell development. Th17 cell suppression should cause a less severe colitis, at least when Th1 cells dominate. In addition to different subpopulations of CLP lymphocytes, we also found fewer CLP lymphocytes in the IL-10 KO/Bcl-2 Tg mice than in the IL-10 KO mice (Figure 5F), suggesting the effect of mucosal integrity on the number of infiltrated CLP lymphocytes. The difference in lymphocyte number should influence disease severity.

\section{Discussion}

In the present study, we demonstrated that suppression of cell death in the intestinal epithelium largely protected the 
development of IBD. Many investigators have considered the causative relationship between epithelial cell death and colitis $^{5,19-21}$ for the following reasons: the number of dying epithelial cells correlates well with the severity of colitis and excessive epithelial cell death seems to disturb the epithelial barrier that allows bacterial invasion, a critical factor for colitis. The latter explanation is conceivable because the intestinal epithelium is a single cell layer that separates submucosal immune cells from luminal components, including food antigens and bacteria. ${ }^{4,5}$ This concept is supported, in part, by a drug-induced acute colitis model, in which Puma (pro-apoptotic protein) knockout mice showed resistance. ${ }^{5,6}$ However, this has not been proved yet in case of chronic colitis. Although several genetic approaches, such as NF- $\kappa \mathrm{B}$-related knockout mice, proposed the possible involvement of epithelial cell death, ${ }^{22-24}$ conclusive evidence using apoptosis molecules is lacking. Moreover, some studies are in disagreement regarding the observations that suppression of epithelial cell apoptosis by caspase inhibition does not maintain the barrier function. ${ }^{4,5}$ Therefore, by using epithelial cell-specific Bcl-2 Tg mice, we attempted to derive a definitive conclusion regarding the role of epithelial cell death on the development of chronic colitis.

We studied IL-10 KO mice as an appropriate model for IBD. As shown in Figure 1, these mice displayed typical features of chronic colitis, including multifocal inflammatory lesions with epithelial hyperplasia and aphthous ulcers. We observed increased epithelial cell death in all inflammatory lesions. Although many studies have focused on epithelial cell apoptosis, we also observed significant necrosis-like cell death. Interestingly, necrosis-like cells contained many bacteria, whereas apoptotic cells did not, suggesting that intracellular bacterial invasion is responsible for necrosis-like death, probably via the production of bacterial products or by autocrine secretion of cytokines. More important, these two forms of cell death were equivalently suppressed by Bcl-2 (Figure 3E). How did Bcl-2 inhibit the appearance of necrosis-like cells? We considered that necrosis-like cells may die through an apoptotic signaling pathway and that the bacterial products may confer the necrosis-like morphological characteristics. However, this idea was incorrect because apoptotic signals, such as cytochrome $c$ release and caspase activation, were not observed. Apoptosis may have aided the induction of necrosis-like cells, possibly through the secretion of inflammatory factors. In this scenario, induction of necrosislike cells might be suppressed by Bcl-2.

Some recent reports have suggested that radioimmunoprecipitation (RIP)-mediated necroptosis plays a role in ileitis. $^{25,26}$ Necroptosis is an apoptosis-independent programed necrosis mediated by RIP1 and RIP3 that is not blocked by Bcl-2. Although necrosis-like cell death in IL-10 $\mathrm{KO}$ mice has the same morphological characteristics as necroptosis, it may be unlikely for the following reasons: necrosis-like cells were efficiently inhibited by Bcl-2, and
Nec-1, a specific inhibitor of necroptosis, did not alter colitis development (data not shown).

Paneth cells, which secrete many antimicrobial factors, play a role in inflammatory bowel diseases. ${ }^{25-27}$ Therefore, their abnormalities activate intestinal bacteria and accelerate inflammation. Atg16 KO mice, which lack the autophagy machinery, develop ileitis because of the functional disturbance of Paneth cells. ${ }^{27}$ In addition, the ileitis of caspase- 8 conditional knockout mice developed by the necroptosis of Paneth cells. ${ }^{25,26}$ Therefore, we examined the number, morphological characteristics, and function of Paneth cells in the small intestines of IL-10 KO and IL-10 KO/Bcl-2 Tg mice. However, we did not observe any abnormalities in the Paneth cells of either type of mouse (Supplemental Figure S6), suggesting that these cells are not involved in the development of IBD in IL-10 KO mice.

Intestinal inflammation develops because of cytokine production from Th1 and Th17 lymphocytes (IL-17/IL-22producing cells). ${ }^{17,18}$ The roles of Th17 lymphocytes are supported by genome-wide association studies indicating the relationship between Th17 differentiation and susceptibility to human $\mathrm{CD} \cdot{ }^{28}$ How does epithelial cell death influence to these cytokine profiles? In case of Th1 cytokines, epithelial cell-specific Bcl-2 had no effect, and the Th1/ Th2 balance was completely shifted to Th1, even in IL-10 $\mathrm{KO} / \mathrm{Bcl}-2 \mathrm{Tg}$ mice, indicating that the Th1 shift was a primary event in IL-10 deficiency. These findings also indicated that a simple Th1 shift is not enough to develop extensive colitis. By contrast, the population of Th17 lymphocytes in IL-10 KO mice was higher than that of WT mice and was markedly suppressed by the epithelialexpressing Bcl-2. The population of Th17 lymphocytes was parallel to disease severity. These data suggested that loss of epithelial integrity activates Th17 cells, leading to severe colitis in IL-10 KO mice.

From the morphological and immunological analyses, we suggest a possible scenario that accounts for the development of colitis: i) low-level infection of bacteria triggers epithelial cell damage; ii) this infection induces mild epithelial cell death together with Th1 cytokines; iii) during this step, intracellular bacteria proliferate intensively and induce increased cell death; iv) increased cell death damages the mucosal barrier, resulting in antigenic exposure of CLP lymphocytes and activation of Th17 lymphocytes; and v) IL17 and bacterial products may facilitate the development of colitis. In this scenario, Bcl-2 seems to protect against this vicious cycle by inhibiting epithelial cell death and maintaining cellular health. Taken together, our results imply that epithelial cell death is an important component of the disorders of chronic colitis.

\section{Acknowledgments}

We thank Akiko Nishimura, Chika Ariga, and Reiko Tsubouchi for technical assistance and Drs. Chu Matsuda, 
Hiroshi Tamagawa, Yasuyuki Kai, Daisuke Kishi, and Hiroshi Kiyono for critical comments.

\section{Supplemental Data}

Supplemental material for this article can be found at http://dx.doi.org/10.1016/j.ajpath.2013.08.012.

\section{References}

1. Bouma G, Strober W: The immunological and genetic basis of inflammatory bowel disease. Nat Rev Immunol 2003, 3:521-533

2. Podolsky DK: Inflammatory bowel disease. N Engl J Med 2002, 347: 417-429

3. Shanahan F: Crohn's disease. Lancet 2002, 359:62-69

4. Marini M, Bamias G, Rivera-Nieves J, Moskaluk CA, Hoang SB, Ross WG, Pizarro TT, Cominelli F: TNF-alpha neutralization ameliorates the severity of murine Crohn's-like ileitis by abrogation of intestinal epithelial cell apoptosis. Proc Natl Acad Sci U S A 2003, $100: 8366-8371$

5. Dirisina R, Katzman RB, Goretsky T, Managlia E, Mittal N, Williams DB, Qiu W, Yu J, Chandel NS, Zhang L, Barrett TA: p53 and PUMA independently regulate apoptosis of intestinal epithelial cells in patients and mice with colitis. Gastroenterology 2011, 141: 1036-1045

6. Qiu W, Wu B, Wang X, Buchanan ME, Regueiro MD, Hartman DJ, Schoen RE, Yu J, Zhang L: PUMA-mediated intestinal epithelial apoptosis contributes to ulcerative colitis in humans and mice. J Clin Invest 2011, 121:1722-1732

7. Lacronique V, Mignon A, Fabre M, Viollet B, Rouquet N, Molina T, Porteu A, Henrion A, Bouscary D, Varlet P, Joulin V, Kahn A: Bcl-2 protects from lethal hepatic apoptosis induced by an anti-Fas antibody in mice. Nat Med 1996, 2:80-86

8. Shimizu S, Eguchi Y, Kamiike W, Funahashi Y, Mignon A, Lacronique V, Matsuda H, Tsujimoto Y: Bcl-2 prevents apoptotic mitochondrial dysfunction by regulating proton flux. Proc Natl Acad Sci U S A 1998, 95:1455-1459

9. Corazza N, Eichenberger S, Eugster HP, Mueller C: Nonlymphocytederived tumor necrosis factor is required for induction of colitis in recombination activating gene (RAG)2(-/-) mice upon transfer of CD4(+)CD45RB(hi) T cells. J Exp Med 1999, 190:1479-1492

10. Kabashima K, Saji T, Murata T, Nagamachi M, Matsuoka T, Segi E, Tsuboi K, Sugimoto Y, Kobayashi T, Miyachi Y, Ichikawa A, Narumiya S: The prostaglandin receptor EP4 suppresses colitis, mucosal damage and CD4 cell activation in the gut. J Clin Invest 2002, 109:883-893

11. Mizushima T, Ito T, Kishi D, Kai Y, Tamagawa H, Nezu R, Kiyono H, Matsuda H: Therapeutic effects of a new lymphocyte homing reagent FTY720 in interleukin-10 gene-deficient mice with colitis. Inflamm Bowel Dis 2004, 10:182-192

12. Kühn R, Löhler J, Rennick D, Rajewsky K, Müller W: Interleukin-10deficient mice develop chronic enterocolitis. Cell 1993, 75:263-274
13. Davidson NJ, Leach MW, Fort MM, Thompson-Snipes L, Kühn R, Müller W, Berg DJ, Rennick DM: T helper cell 1-type CD4+ T cells, but not B cells, mediate colitis in interleukin 10-deficient mice. J Exp Med 1996, 184:241-251

14. Rennick DM, Fort MM, Davidson NJ: Studies with IL-10-/- mice: an overview. J Leukoc Biol 1997, 61:389-396

15. Aluwihare AP: Electron microscopy in Crohn's disease. Gut 1971, 12 509-518

16. Davidson NJ, Hudak SA, Lesley RE, Menon S, Leach MW, Rennick DM: IL-12, but not IFN-gamma, plays a major role in sustaining the chronic phase of colitis in IL-10-deficient mice. J Immunol 1998, 161:3143-3149

17. Brand S: Crohn's disease: Th1, Th17 or both? the change of a paradigm: new immunological and genetic insights implicate Th17 cells in the pathogenesis of Crohn's disease. Gut 2009, 58:1152-1167

18. Strober W, Fuss IJ: Proinflammatory cytokines in the pathogenesis of inflammatory bowel diseases. Gastroenterology 2011, 140:1756-1767

19. Iwamoto M, Koji T, Makiyama K, Kobayashi N, Nakane PK: Apoptosis of crypt epithelial cells in ulcerative colitis. J Pathol 1996, 180:152-159

20. Hagiwara C, Tanaka M, Kudo H: Increase in colorectal epithelial apoptotic cells in patients with ulcerative colitis ultimately requiring surgery. J Gastroenterol Hepatol 2002, 17:758-764

21. Di Sabatino A, Ciccocioppo R, Luinetti O, Ricevuti L, Morera R, Cifone MG, Solcia E, Corazza GR: Increased enterocyte apoptosis in inflamed areas of Crohn's disease. Dis Colon Rectum 2003, 46: $1498-1507$

22. Sträter J, Wellisch I, Riedl S, Walczak H, Koretz K, Tandara A, Krammer PH, Möller P: CD95 (APO-1/Fas)-mediated apoptosis in colon epithelial cells: a possible role in ulcerative colitis. Gastroenterology 1997, 113:160-167

23. Steinbrecher KA, Harmel-Laws E, Sitcheran R, Baldwin AS: Loss of epithelial RelA results in deregulated intestinal proliferative/apoptotic homeostasis and susceptibility to inflammation. J Immunol 2008, 180: 2588-2599

24. Nenci A, Becker C, Wullaert A, Gareus R, van Loo G, Danese S, Huth M, Nikolaev A, Neufert C, Madison B, Gumucio D, Neurath MF Pasparakis M: Epithelial NEMO links innate immunity to chronic intestinal inflammation. Nature 2007, 446:557-561

25. Günther C, Martini E, Wittkopf N, Amann K, Weigmann B, Neumann H, Waldner MJ, Hedrick SM, Tenzer S, Neurath MF, Becker C: Caspase-8 regulates TNF-alpha-induced epithelial necroptosis and terminal ileitis. Nature 2011, 477:335-339

26. Welz PS, Wullaert A, Vlantis K, Kondylis V, Fernández-Majada V, Ermolaeva M, Kirsch P, Sterner-Kock A, van Loo G, Pasparakis M: FADD prevents RIP3-mediated epithelial cell necrosis and chronic intestinal inflammation. Nature 2011, 477:330-334

27. Cadwell K, Liu JY, Brown SL, Miyoshi H, Loh J, Lennerz JK, Kishi C, Kc W, Carrero JA, Hunt S, Stone CD, Brunt EM, Xavier RJ, Sleckman BP, Li E, Mizushima N, Stappenbeck TS, Virgin HW: A key role for autophagy and the autophagy gene Atg1611 in mouse and human intestinal Paneth cells. Nature 2008, 456:259-263

28. Barrett JC, Hansoul S, Nicolae DL, Cho JH, Duerr RH, Rioux JD, et al: Genome-wide association defines more than 30 distinct susceptibility loci for Crohn's disease. Nat Genet 2008, 40:955-962 